\title{
3-D Poro-Elastoplastic Model for Short-Crested Wave-Induced Pore Pressures in a Porous Seabed
}

\author{
Z. S. Wong ${ }^{1}$, C. C. Liao $^{1}$ and D. S. Jeng ${ }^{1,2, *}$ \\ ${ }^{1}$ Center for Marine Geotechnical Engineering Research, State Key Laboratory of Ocean Engineering, Department of \\ Civil Engineering, Shanghai Jiao Tong University, Shanghai, 200240 China \\ ${ }^{2}$ Griffith School of Engineering, Griffith University Gold Coast Campus, Queensland 4222, Australia
}

\begin{abstract}
In this paper, a three-dimensional poro-elastoplastic model for the short-crested wave-induced pore pressures in a porous seabed is presented. Unlike the previous models, both elasticity and plasticity of seafloor are considered in the present model. This study considers the effects of wave and soil characteristics on the pore pressures and was validated with the previous wave experiment data. As the numerical analysis shows, higher value of plastic parameter leads to a faster residual pore pressure accumulation, which is closely related to the occurrence of seabed liquefaction. In particular, at the dissipation stage, residual pore pressure sharply decreases when enlarging plastic parameter $\beta$, which dominates the velocity of accumulation of plastic volumetric strain.
\end{abstract}

Keywords: Elasto-plastic soil, pore pressure, short-crested wave.

\section{INTRODUCTION}

In recent years, it has been reported in the literature that numerous coastal and ocean structures have been damaged by the wave-induced seabed response, rather than construction deficiencies [1-3]. Benefiting from this awareness among geotechnical engineers, intensive research of seabed dynamic reactions with external loads (such as waves, winds, and earthquakes etc.) could go further.

As shown in Fig. (1), when a progressive wave arrives obliquely at a vertical wall, it would be reflected from the structure into a new direction, which is normal to the original incoming direction, resulting into a short-crested wave system. This kind of wave would double the wave height at crest intersections, complicate the pattern of seawaterparticle motions, and thus reinforce the seabed instability [4]. While propagating in the ocean, waves generate significant dynamic fluctuation on sea floor, which in turn evokes pore water pressures and effective stresses within the sea floor. With accumulating pore pressure and diminishing vertical effective stress, part of the seabed might become unsteady or even liquefied. Once liquefaction occurs, the soil skeleton which is referred to the soil particles would lose resistance to any shear stress, and are likely to be carried away as a heavy fluid by any prevailing seabed surface current, which leads to the failure of load bearing, resulting in the destruction of the whole structure [4]. Therefore, the predictions of wave-

*Address correspondence to this author at the Center for Marine Geotechnical Engineering Research, State Key Laboratory of Ocean Engineering, Department of Civil Engineering, Shanghai Jiao Tong University, Shanghai, 200240 China; E-mail: d.jeng@griffith.edu.au

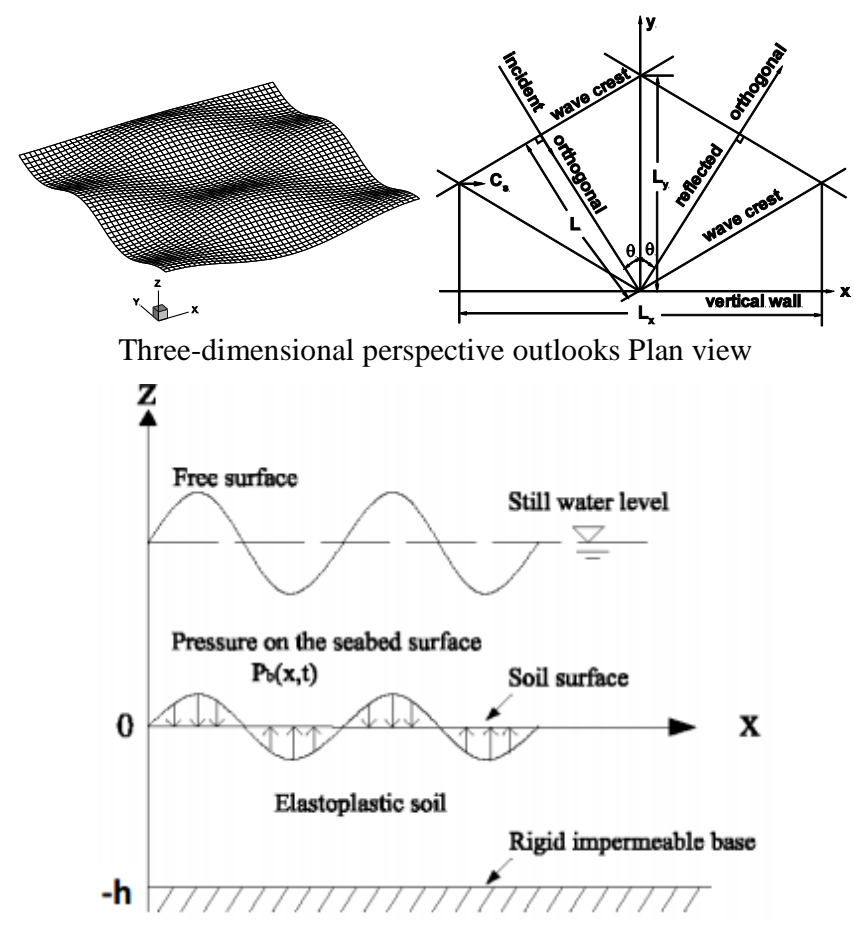

Vertical profile of waves traveling over a porous seafloor

Fig. (1). Sketch of a short-crested wave system.

induced liquefaction and shear failure are essential in the design of marine infrastructures.

Generally speaking, two mechanisms of wave-induced pore pressure have been observed in the field measurements 
and laboratory experiments (see Fig. 2). The first mechanism is resulted from the transient or oscillatory excess pore pressure and is accompanied by attenuation of amplitude and lag of phase [5]. This mechanism displays elastic aspect of the seabed sediments, and can be treated as a periodical response towards short-crested waves. While the second mechanism caused by the residual or progressive feature of marine sediments is quite different, it comprises the accumulation and dissipation process. Explicitly, the contraction of the soil under surface dynamic cyclic loading makes the accumulation of residual pore pressure become possible [6].

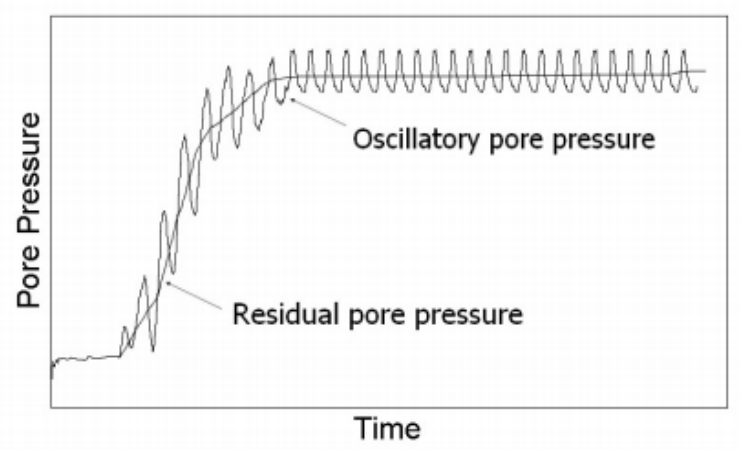

Fig. (2). Mechanism of oscillatory and residual soil response.

Considerable studies for wave-induced momentary liquefaction, caused by oscillatory pore pressure, have been accomplished in the last several decades [7, 8]. Based on the conventional Biot's consolidation theory [9], this field has been further extended to a new level. For example, seabed is treated as a non-homogeneous medium, and soil behavior can be cross-anisotropic [10]. The mechanism of residual pore pressure has also accessed progresses. Seed and Rahman [5] established a simple 1-D finite element model by taking into account of distribution of periodical shear stresses in the soil profile. However, their solution regarding of pore pressure accumulation was formulated without considering seabed plasticity and was limited to only onedimensional. Sekiguchi et al. [11] proposed a poroelastoplastic model for a shallow soil layer to analyze standing wave-induced liquefaction using a Laplace transformation. Since then, the property of seafloor plasticity in wave-seabed interactions has been largely emphasized owing to the practice of numerous centrifuge tests [12]. Later, several numerical models for post-liquefaction and densification research in marine sediments were developed [12]. Moreover, it is worthwhile to mention the 2D model proposed by Liao at el. [13], which creatively concentrates on the effect of seabed plasticity in the wave seabed interactions.

In this paper, a 3D poro-elastoplastic model is developed, and then it will be verified with the previous experimental data. Moreover, a parametric study will be conducted to examine the influences of wave and soil characteristics on both oscillatory and residual pore pressure in marine sediments.

\section{THEORETICAL FORMULATIONS}

The proposed poro-elastoplastic model integrates both elasticity and plasticity of marine sediments. Herein, the present model can definitely provide a better prediction of the potential of the wave-induced seabed instability than previous elastic model, which neglects plasticity of marine sediments, and cannot be applied to large deformation.

In the model, when a short-crested wave propagates over the seabed surface, it might induce fluctuations on the seabed surface, arousing both transient and residual pore pressure.

$\mathrm{p}=\mathrm{p}_{1}+\mathrm{p}_{2}$

According to the previous research, the average transient pore pressure $p_{1}$ over any wave cycle is zero, while residual pore pressure $\mathrm{p}_{2}$, which mainly stems from contractive behavior of soil, would develop along with wave cycles.

\subsection{Oscillatory Component}

Biot's consolidation equation is generally accepted to govern the flow of compressible pore fluid in a porous medium. For a three-dimensional case, treating the porous sea floor as hydraulically isotropic, the governing equation [9] can be expressed as

$\frac{\partial^{2} \mathrm{p}_{1}}{\partial \mathrm{x}^{2}}+\frac{\partial^{2} \mathrm{p}_{1}}{\partial \mathrm{y}^{2}}+\frac{\partial^{2} \mathrm{p}_{1}}{\partial \mathrm{z}^{2}}-\frac{\gamma_{\mathrm{w}} \mathrm{n}_{\mathrm{s}} \beta}{\mathrm{k}_{\mathrm{s}}} \frac{\partial \mathrm{p}_{1}}{\partial \mathrm{t}}+\frac{\gamma_{\mathrm{w}}}{\mathrm{k}_{\mathrm{s}}} \frac{\partial \varepsilon_{1}}{\partial \mathrm{t}}=0$

where $p_{1}$ is the wave-induced oscillatory pore pressure, $n_{s}$ is the soil porosity, $\mathrm{k}_{\mathrm{s}}$ is the permeability of sea floor, and $\gamma_{w}$ is the unit weight of pore water. The elastic volume strain of seabed matrix $\varepsilon_{1}$ and pore fluid compressibility $\beta$ are defined as:

$\varepsilon_{1}=\frac{\partial \mathrm{u}}{\partial \mathrm{x}}+\frac{\partial \mathrm{v}}{\partial \mathrm{y}}+\frac{\partial \mathrm{w}}{\partial \mathrm{z}}$

$\beta=\frac{1}{\mathrm{~K}_{\mathrm{w}}}+\frac{1-\mathrm{S}_{\mathrm{r}}}{\mathrm{P}_{\mathrm{wo}}}$,

where $\mathrm{u}, \mathrm{v}$ and $\mathrm{w}$ are the soil displacements in the $x, y$, and $z$ direction, respectively. $\mathrm{K}_{\mathrm{w}}$ is the true bulk modulus of water elasticity, which is suggested to take $1.9 \times 10^{9} \mathrm{~N} / \mathrm{m}^{2}$ [14] as an approximation value of laboratory data. $\mathrm{P}_{\mathrm{wo}}$ is the absolute water pressure, and $S_{r}$ is the degree of seabed saturation. Explicitly, when $S_{r}=1$, it means the soil skeleton is completely air-free, pore fluid compressibility can be conveyed as $\beta=\frac{1}{\mathrm{~K}_{\mathrm{w}}}$.

The equation for overall equilibrium, related to the effective stress and pore pressure, can be written as

$\frac{\partial \sigma_{\mathrm{x}}^{\prime}}{\partial \mathrm{x}}+\frac{\partial \tau_{\mathrm{xy}}}{\partial \mathrm{y}}+\frac{\partial \tau_{\mathrm{xz}}}{\partial \mathrm{z}}=-\frac{\partial \mathrm{p}_{1}}{\partial \mathrm{x}}$

$\frac{\partial \tau_{\mathrm{xy}}}{\partial \mathrm{x}}+\frac{\partial \sigma_{\mathrm{y}}^{\prime}}{\partial \mathrm{y}}+\frac{\partial \tau_{\mathrm{yz}}}{\partial \mathrm{z}}=-\frac{\partial \mathrm{p}_{1}}{\partial \mathrm{y}}$

$\frac{\partial \tau_{\mathrm{xz}}}{\partial \mathrm{x}}+\frac{\partial \tau_{\mathrm{yz}}}{\partial \mathrm{y}}+\frac{\partial \sigma_{\mathrm{z}}^{\prime}}{\partial \mathrm{z}}=-\frac{\partial \mathrm{p}_{1}}{\partial \mathrm{z}}$

where $\sigma_{\mathrm{x}}^{\prime}, \sigma_{\mathrm{y}}^{\prime} \sigma_{\mathrm{z}}^{\prime}, \tau_{\mathrm{xz}}, \tau_{\mathrm{yz}}, \tau_{\mathrm{xy}}$, are the effective normal stress in the $x$-, $y$ - and $z$-directions, respectively; and $\quad x z, y z$ and $x y$ are shear stresses. 
Based on the generalized Hooke's law, the relationships between elastic incremental effective stresses and soil displacements are given by

$$
\begin{aligned}
\sigma_{\mathrm{x}}^{\prime} & =2 \mathrm{G}\left[\frac{\partial \mathrm{u}}{\partial \mathrm{x}}+\frac{\mu}{1-2 \mu} \varepsilon_{1}\right] \\
\sigma_{\mathrm{y}}^{\prime} & =2 \mathrm{G}\left[\frac{\partial \mathrm{v}}{\partial \mathrm{y}}+\frac{\mu}{1-2 \mu} \varepsilon_{1}\right] \\
\sigma_{\mathrm{z}}^{\prime} & =2 \mathrm{G}\left[\frac{\partial \mathrm{w}}{\partial \mathrm{z}}+\frac{\mu}{1-2 \mu} \varepsilon_{1}\right] \\
\tau_{\mathrm{xz}} & =\tau_{\mathrm{zx}}=G\left[\frac{\partial \mathrm{u}}{\partial \mathrm{z}}+\frac{\partial \mathrm{w}}{\partial \mathrm{x}}\right] \\
\tau_{\mathrm{yz}} & =\tau_{\mathrm{zy}}=G\left[\frac{\partial \mathrm{v}}{\partial \mathrm{z}}+\frac{\partial \mathrm{w}}{\partial \mathrm{y}}\right] \\
\tau_{\mathrm{xy}} & =\tau_{\mathrm{yx}}=G\left[\frac{\partial \mathrm{u}}{\partial \mathrm{y}}+\frac{\partial \mathrm{v}}{\partial \mathrm{x}}\right]
\end{aligned}
$$

in which the shear modulus $G$ is connected with Young's modulus E via the Poisson's ratio $\mu$ in the form of $\frac{E}{2(1+\mu)}$.

Substituting (8)-(13) into (5)-(7), the force equilibrium equation are transformed as

$$
\begin{aligned}
& \mathrm{G} \nabla^{2} \mathrm{u}+\frac{\mathrm{G}}{1-2 \mu} \frac{\partial \varepsilon_{1}}{\partial \mathrm{x}}=-\frac{\partial \mathrm{p}_{1}}{\partial \mathrm{x}} \\
& \mathrm{G} \nabla^{2} \mathrm{v}+\frac{\mathrm{G}}{1-2 \mu} \frac{\partial \varepsilon_{1}}{\partial \mathrm{y}}=-\frac{\partial \mathrm{p}_{1}}{\partial \mathrm{y}} \\
& \mathrm{G} \nabla^{2} \mathrm{w}+\frac{\mathrm{G}}{1-2 \mu} \frac{\partial \varepsilon_{1}}{\partial \mathrm{z}}=-\frac{\partial \mathrm{p}_{1}}{\partial \mathrm{z}}
\end{aligned}
$$

in the $\mathrm{x}, \mathrm{y}$ and $\mathrm{z}$ directions, respectively.

\subsection{Residual Component}

In this section, the residual component of pore water pressure is considered via a relatively simple but a workable plastic constitutive relation with cumulative contraction of soil, which directly attributes to the residual pore water pressure accumulation process. Through the method of deduction, the formula concerning residual pore pressure can be derived to $3 \mathrm{D}$, which is a generalization of the earlier $2 \mathrm{D}$ situation completed by Liao et al. [13].

With the presentations launched above, it is generally accepted to divide the total volumetric strain increment of seafloor sediments exposed to cyclic wave loading into an elastic part $\varepsilon_{1}$ and a plastic part $\varepsilon_{2}$, that is to say

$\varepsilon=\varepsilon_{1}+\varepsilon_{2}$

Since the elastic volumetric strain increment $d \varepsilon_{1}$ could be expressed via the increment of vertical effective over burden $\mathrm{d} \sigma_{\mathrm{z}}^{\prime}$ in the form of $\mathrm{d} \varepsilon_{1}=m_{v} \mathrm{~d} \sigma_{\mathrm{z}}^{\prime}$, in which $m_{v}$ is the coefficient of soil volume compressibility. Thus,

$\frac{\partial \varepsilon}{\partial \mathrm{t}}=\frac{\partial \varepsilon_{1}}{\partial \mathrm{t}}+\frac{\partial \varepsilon_{2}}{\partial \mathrm{t}}=m_{v} \frac{\partial \sigma_{\mathrm{z}}^{\prime}}{\partial \mathrm{t}}+\frac{\partial \varepsilon_{2}}{\partial \mathrm{t}}$

The storage equation regarding to a deformable soil can be conveyed as

$\frac{\partial \varepsilon}{\partial \mathrm{t}}=-\frac{\mathrm{k}_{\mathrm{s}}}{\gamma_{\mathrm{w}}}\left(\frac{\partial^{2} \mathrm{p}}{\partial \mathrm{x}^{2}}+\frac{\partial^{2} \mathrm{p}}{\partial \mathrm{y}^{2}}+\frac{\partial^{2} \mathrm{p}}{\partial \mathrm{z}^{2}}\right)$

Combining the equations above, we obtain $m_{v}\left(\frac{\partial \sigma_{\mathrm{z}}}{\partial \mathrm{t}}-\frac{\partial \mathrm{p}}{\partial \mathrm{t}}\right)+\frac{\partial \varepsilon_{2}}{\partial \mathrm{t}}=-\frac{\mathrm{k}_{\mathrm{s}}}{\gamma_{\mathrm{w}}}\left(\frac{\partial^{2} \mathrm{p}}{\partial \mathrm{x}^{2}}+\frac{\partial^{2} \mathrm{p}}{\partial \mathrm{y}^{2}}+\frac{\partial^{2} \mathrm{p}}{\partial \mathrm{z}^{2}}\right)$

As the time average $\bar{\sigma}_{\mathrm{z}}$ and $\overline{\mathrm{p}}_{1}$ over any wave cycle can be simply taken as zero, we acquired the residual pore pressure governing equation

$\frac{\partial \mathrm{p}_{2}}{\partial \mathrm{t}}=\frac{1}{m_{v}} \frac{\partial \varepsilon_{2}}{\partial \mathrm{t}}+\frac{\mathrm{k}_{\mathrm{s}}}{m_{v} \gamma_{\mathrm{w}}}\left(\frac{\partial^{2} p_{2}}{\partial \mathrm{x}^{2}}+\frac{\partial^{2} p_{2}}{\partial \mathrm{y}^{2}}+\frac{\partial^{2} p_{2}}{\partial \mathrm{z}^{2}}\right)$

Owing to the plasticity of soil, it is quite reasonable that sands with loose to medium density are likely to exhibit a decrease of accumulative volume while exposed to repetitive wave loading, which is principally linked to shear stress $\tau$ and loading cycle $\psi$ as reported by Sekiguchi et al. [11]. For loosely packed sand, the plastic volumetric strain $\varepsilon_{2}$ under fixed amplitude of periodical loading at a specific point should be expressed as

$\varepsilon_{2}(\psi, \phi)=\varepsilon_{2}^{\infty}\left(1-\mathrm{e}^{-\beta \psi}\right)$

where $\beta$ is a material parameter dominating the velocity of accumulation of plastic volumetric strain, as mentioned above, $\psi$ is the wave repetitions, equivalent to $\mathrm{t} / \mathrm{T}$, While $\varepsilon_{2}^{\infty}$ represents the plastic volumetric strain when $\psi$ ultimately approaches infinity.

Based on the foundation of the hypothesis, for a threedimensional case, the cyclic stress ratio can be expressed as

$\phi(\mathrm{x}, \mathrm{y}, \mathrm{z})=\frac{\sqrt{\tau_{\mathrm{xy}}^{2}+\tau_{\mathrm{yz}}^{2}+\tau_{\mathrm{xz}}^{2}}}{\sigma_{0}}$

where $\sigma_{0}$ stands for the preliminary vertical effective stress defined by

$\sigma_{0}=-\left(\gamma_{\mathrm{s}}-\gamma_{\mathrm{w}}\right) \mathrm{z}$

Since $\mathrm{z} \leq 0$, on the basis of Sekiguchi's previous conclusion [11], $\varepsilon_{2}^{\infty}$ is also interconnected with material parameter $\alpha$ and $\mathrm{R}$ rather than solely $\phi$, in the form of $\varepsilon_{2}^{\infty}=$ $\mathrm{R}\left(\mathrm{e}^{\alpha \phi}\right)$. Therefore we rewrite the expression of plastic volumetric strain as

$\varepsilon_{2}=\mathrm{R}\left(\mathrm{e}^{\alpha \phi}-1\right)\left(1-\mathrm{e}^{-\beta \psi}\right)$

Introducing $\psi$ and $\phi$ to the initial residual pore water pressure governing equation, $\omega$ is the wave frequency defined by $\omega=\frac{2 \pi}{\mathrm{T}}$, we obtain the final version.

$\frac{\partial \mathrm{p}_{2}}{\partial \psi}=\frac{2 \pi \mathrm{k}_{\mathrm{s}}}{\omega m_{v} \gamma_{\mathrm{w}}}\left(\frac{\partial^{2} \mathrm{p}_{2}}{\partial \mathrm{x}^{2}}+\frac{\partial^{2} \mathrm{p}_{2}}{\partial \mathrm{y}^{2}}+\frac{\partial^{2} \mathrm{p}_{2}}{\partial \mathrm{z}^{2}}\right)+\frac{1}{m_{v}} \mathrm{R} \beta \mathrm{e}^{-\beta \psi}\left(\mathrm{e}^{\alpha \phi}-1\right)$

On the right hand side of (26), it is worthy of attention that the first term shows the accumulation and dissipation rate, while the second term indicates the influence of cyclic loading on the cumulating process of residual pore pressure.

For a normal consolidation problem, the value of the constrained modulus $\mathrm{M}$, defined by $\mathrm{M}=\frac{1}{m_{v}}$, raises linearly with increasing effective confining pressure, that is

$\mathrm{M}=-\frac{\mathrm{z}}{\mathrm{h}} \mathrm{M}_{\mathrm{h}},-\mathrm{h} \leq \mathrm{z} \leq 0$

where $M_{h}$ represents the value of $M$ at the bottom of the seafloor. 


\subsection{Boundary Conditions}

For a homogeneous seabed, with appropriate boundary conditions, mathematical solutions for the wave-induced soil response can be derived. Basically, two of these are required for a seafloor of finite thickness.

It is commonly acceptable that both vertical effective over burden and shear stresses vanish at the surface of the seabed, where $\mathrm{z}=0$.

$\sigma_{\mathrm{z}}^{\prime}=\tau_{\mathrm{xy}}=\tau_{\mathrm{yz}}=0$

At the surface of the seabed, oscillatory pore pressure is equivalent to the short-crested wave pressure [15].

$\mathrm{p}_{1}=\mathrm{p}_{\mathrm{b}}=\frac{\gamma_{\mathrm{w}} \mathrm{H}}{2 \cosh \mathrm{kd}} \cos (\mathrm{nky}) \cos (\mathrm{mkx}-\omega \mathrm{t})$

where $\mathrm{H}$ is the short-crested wave height, $\mathrm{k}$ is the wave number of the incident and reflected wave (equals $2 \pi / L$, in which $L$ is the wavelength), $d$ is the water depth above the seafloor surface. Two wave obliquity parameters $m$ and $n$ are connected via the equation $\mathrm{m}^{2}+\mathrm{n}^{2}=1$ (where $\mathrm{m}=\sin \theta$ and $\mathrm{n}=\cos \theta), \theta$ is the wave angle between the incident waves and normal of the vertical wall.)

For the residual pore water pressure $\left(\mathrm{p}_{2}\right)$ at the seabed surface, it is as nil, that is $\mathrm{p}_{2}=0$.

For the seafloor of finite thickness, no displacements and vertical flow were observed at the impermeable rigid bottom, and the diversification velocity of residual pore pressure versus depth $\mathrm{z}$ maintains constant.

$$
\mathrm{u}=\mathrm{v}=\mathrm{w}=0, \frac{\partial \mathrm{p}_{1}}{\partial \mathrm{z}}=\frac{\partial \mathrm{p}_{2}}{\partial \mathrm{z}}=0
$$

In this study, we have successfully established a poroelastopalstic model in finite element software COMSOL (version 4.3a), which is proficient in coupling multi-physics environments. Specifically, first, we set the present model in three-dimensional seabed environment, and select Partial Differential Equations Interface as the numerical solver; second, input all the parameters and variables (some are expressed as relations) derived from the theoretical part in the text; then build up corresponding constraints or periodical conditions at the interface. Eventually, only the previous preparing procedures are appropriately completed, could we start to run the case and obtain the numerical solutions.

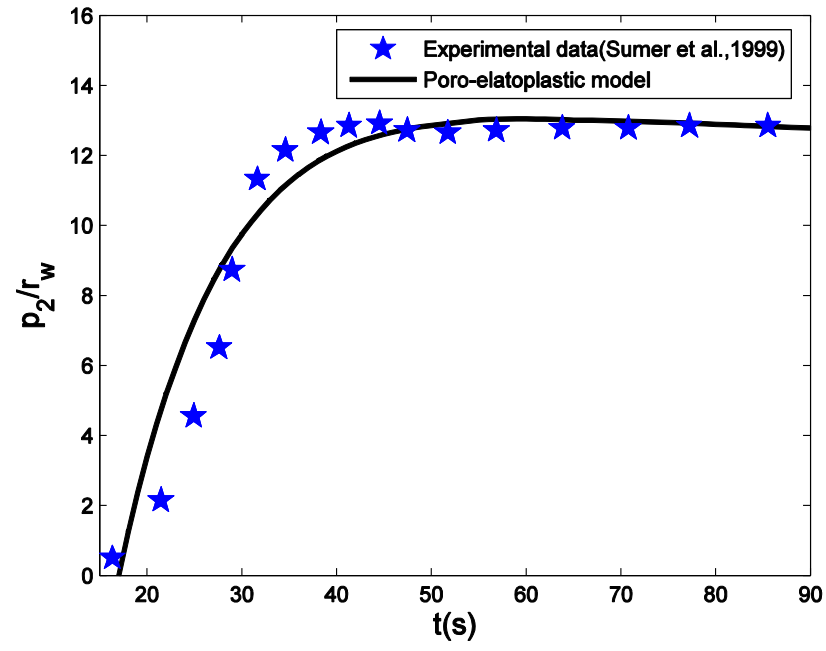

Fig. (3). Comparison of residual pore pressure with Sumer's wave experiment data [16].

\section{VALIDATION}

Since the present three-dimensional numerical model is new and the relevant experimental investigations are not available, it is necessary to verify them with previous experiment data, which mainly include the wave experiment carried out by Sumer et al. [16].

Sumer et al. [16] have accomplished considerable laboratory experiments in a relatively silt soil, and have acquired several meaningful results regarding pore pressure accumulation under progressive wave loading. They confirmed that pore water pressure indeed has an accumulation tendency as wave propagates, and liquefaction would certainly emerge when wave height exceeds a certain value. The parameters related to wave and soil characteristics are tabulated below in Table $\mathbf{1}$, and the corresponding results are plotted in Fig. (3). As shown in the figure, the present model resembles Sumer's experimental data in trend, and these two curves are almost overlapped, which indicates the feasibility of our theory to simulate three-dimensional wave-seabed interactions. It is noted that there are some differences between the present model and experimental data [16] at the beginning stage of pore pressure build up, although the present results agree well with the experimental data after the

Table 1. Input data for the experiment (Sumer et al., 1999).

\begin{tabular}{|c|c|c|c|}
\hline \multicolumn{5}{|c|}{ Wave Variables } \\
\hline \hline Wave Period $(\mathrm{T})$ & Wave Length $(\mathrm{L})$ & Wave Depth $(\mathrm{d})$ & Wave Height $(\mathrm{H})$ \\
\hline $1.6 \mathrm{~s}$ & $2.89 \mathrm{~m}$ & $0.42 \mathrm{~m}$ & $0.166 \mathrm{~m}$ \\
\hline \multicolumn{2}{|c|}{ Soil Variables } \\
\hline \hline Seabed Thickness $(\mathrm{h})$ & Permeability $\left(\mathrm{k}_{\mathrm{s}}\right)$ & Degree of Saturation $\left(\mathrm{S}_{\mathrm{r}}\right)$ & Soil Porosity $\left(\mathrm{n}_{\mathrm{s}}\right)$ \\
\hline $0.165 \mathrm{~m}$ & $5.37 \times 10^{-7} \mathrm{~m} / \mathrm{s}$ & 1 & 0.35 \\
\hline Poisson's Ratio $(\eta)$ & Plastic Parameter $(\alpha)$ & Plastic Parameter $(\beta)$ & Plastic Parameter $(\mathrm{R})$ \\
\hline 0.41 & 55 & 0.1 & $4.6 \times 10^{-4}$ \\
\hline
\end{tabular}


residual pore pressures built up. Possible reasons for these differences are: (i) the assumption of the present model is poro-elastoplastci model, in which the soil plastic behavior was used to describe the pore pressures when the loading is applied, but elastic soil behavior is applied for the un-loading procedure; (ii) since the soil plastic parameters were not measured during the experiments, we can only use the values obtained from other researches, which may cause some differences between the experimental data and the present model.

\section{RESULTS AND DISCUSSIONS}

In this section, both oscillatory and residual pore water pressures are studied to explore the inherent laws versus relevant soil and wave parameters, meanwhile high attention was paid to the occurrence of seabed liquefaction. Explicitly, we select a specific point $(\mathrm{x}, \mathrm{y}, \mathrm{z})=(0,0,-2) m$ to analyze the corresponding interrelationships among these variables. Except the target wave characteristics $(\theta, \mathrm{H} / \mathrm{L})$ and soil characteristics $\left(\mathrm{k}, \mathrm{S}_{\mathrm{r}}\right)$, other input data for the numerical examples are given in Table 2.

\subsection{Effects of Wave characteristics}

In a short-crested wave system, one of the main variables to determine water particle motion and pressure fluctuation at the seabed surface is the angle between the orthogonal and the normal to the wall (referring to Fig. 1), or alternatively between crest alignments and the wall itself [17].

Fig. (4) illustrates the wave-induced soil response including oscillatory and residual pore pressures, effective

Table 2. Input data of numerical examples.

\begin{tabular}{|c|c|c|c|}
\hline \multicolumn{4}{|c|}{ Wave Characteristics } \\
\hline Wave Period (T) & Wave Length (L) & Wave Depth (d) & Wave Height $(\mathrm{H})$ \\
\hline $10 \mathrm{~s}$ & $111.7 \mathrm{~m}$ & $16 \mathrm{~m}$ & $5 \mathrm{~m}$ or Various \\
\hline \multicolumn{4}{|c|}{ Soil characteristics } \\
\hline Seabed Thickness (h) & Permeability $\left(\mathrm{k}_{\mathrm{s}}\right)$ & Degree of Saturation $\left(\mathrm{S}_{\mathrm{r}}\right)$ & Soil Porosity $\left(\mathrm{n}_{\mathrm{s}}\right)$ \\
\hline $50 \mathrm{~m}$ & $1.0 \times 10^{-4} \mathrm{~m} / \mathrm{s}$ or various & 1 or various & 0.3 \\
\hline Poisson’s Ratio ( $)$ & Plastic Parameter $(\alpha)$ & Plastic Parameter $(\beta)$ & Plastic Parameter (R) \\
\hline 0.35 & 55 or Various & 0.1 or Various & $4.6 \times 10^{-5}$ or Various \\
\hline
\end{tabular}

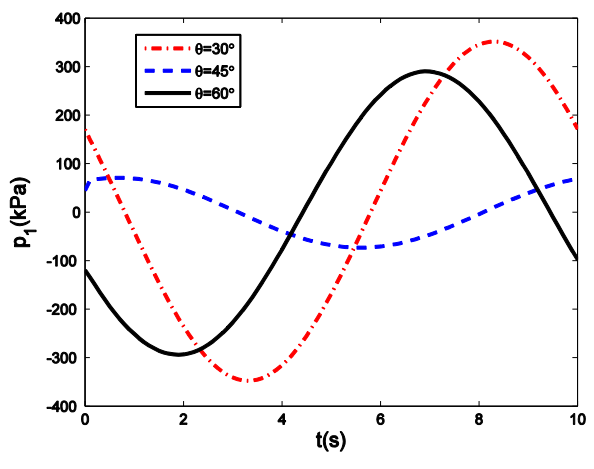

(a) Oscillatory pore pressure

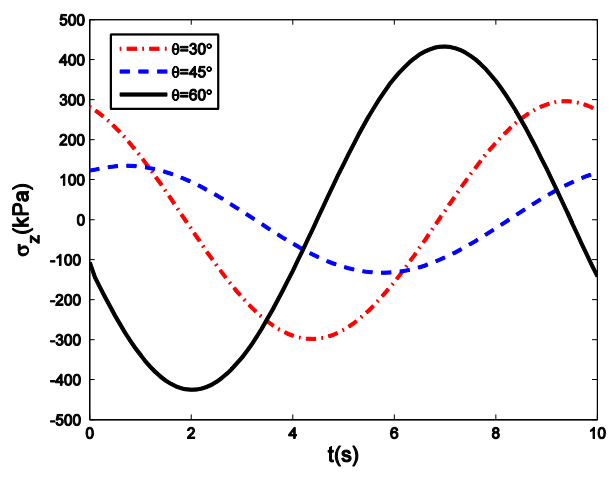

(c) Vertical effective normal stress

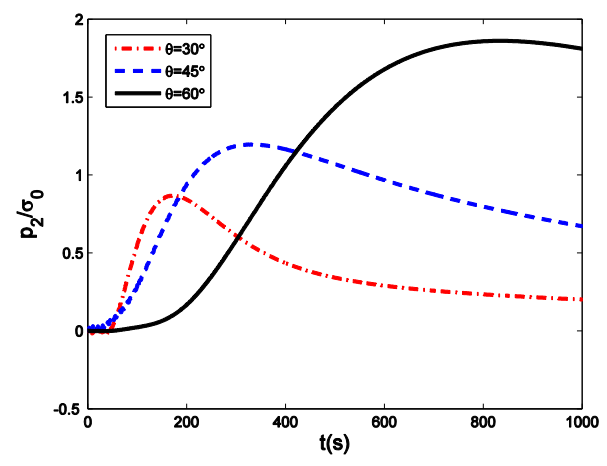

(b) Residual pore pressure

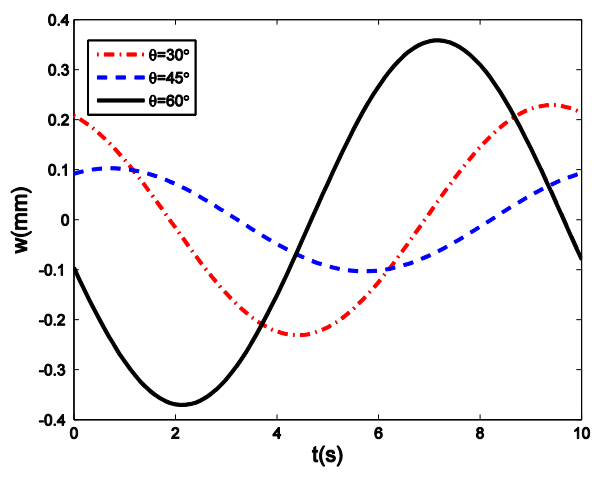

(d) Vertical soil displacements

Fig. (4). Effects of incident angle on wave-seabed interaction at point $(x, y, z)=(0,0,-2) m$. 
normal stresses and soil displacements with various wave obliquity $(\theta)$. In this example, three incident wave angles, $30^{\circ}, 45^{\circ}$, and $60^{\circ}$ are used. As shown in the figure, when $\theta=45^{\circ}$, their amplitudes of soil response variables appear to be the minimum. Particularly, while $\theta=45^{\circ}$, oscillatory pore pressure fluctuate slightly, and might cause the minimum damage to ocean structures, since the liquefaction depth is relatively lower. On the basis of the result, coastal engineers could design the ocean structure in a specific direction to against the attack of storms, even hurricanes according to the perennial scientific observation of ocean wave propagating orientation.

Another important parameter affecting wave-seabed interactions is the wave steepness $(\mathrm{H} / \mathrm{L})$. With the wave steepness ranging from 0.01 to 0.05 , the wave-induced soil responses are presented in Fig. (5). As seen in the picture, when the short-crested wave grows steeper, all soil response variables, like displacement, effective stress, and oscillatory pore pressure are getting larger. Especially, at the wave crests, the steepest wave has the maximum displacement, vertical stress and oscillatory pore pressure. However, it is totally another thing while at wave troughs, where the steepest wave has the minimum displacement, vertical stress and oscillatory pore pressure.

The liquefaction criterion, $\mathrm{p}_{2} / \sigma_{0}=1$ [4], has been commonly used to determine if the seabed is liquefied or not.
In such a condition, the seabed would lose resistance to shear and vertical overload brought about by shot-crested wave. As shown in Fig. (5b), there is no liquefaction occurred at a specific point (in this case $\mathrm{z}=-2 \mathrm{~m}$ ) below the seabed surface for a small wave $(\mathrm{H} / \mathrm{L}=0.01)$, while the liquefaction occurs at the cases of $\mathrm{H} / \mathrm{L}=0.03$ and 0.05 . As shown in the figure, the liquefaction occurs at about $20^{\text {th }}$ wave cycles (see Fig. (5b)).

\subsection{Effects of Soil Characteristics}

Various soil parameters would affect wave-induced soil response. Here, we consider two parameters, seabed permeability and soil saturation, which were reported to have significant effects of the soil response.

The permeability of soil is the measurement of how rapidly fluid is transmitted through the voids between soil grains. In this study, we particularly concentrated on isotropic soil $\left(\mathrm{k}_{\mathrm{x}}=\mathrm{k}_{\mathrm{y}}=\mathrm{k}_{\mathrm{z}}=\mathrm{k}_{\mathrm{s}}\right)$ ranging from coarse sand $\left(\mathrm{k}_{\mathrm{s}}=1.0 \times 10^{-2} \mathrm{~m} / \mathrm{s}\right)$ to fine sand $\left(\mathrm{k}_{\mathrm{s}}=1.0 \times 10^{-4} \mathrm{~m} / \mathrm{s}\right)$.

As illustrated in Fig. (6), soil permeability barely has any influence on vertical displacement and stress, but it has a significant impact on oscillatory pore pressure, which deceases while the soil gets crude. That is to say, the smaller the grain size is, the higher oscillatory pore pressure might be, since it becomes much more difficult to dissipate in fine sand than in coarse sand. Moreover, residual pore water

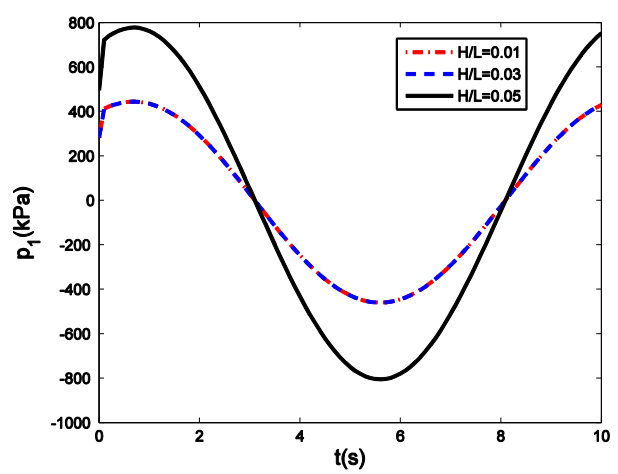

(a) Oscillatory pore pressur

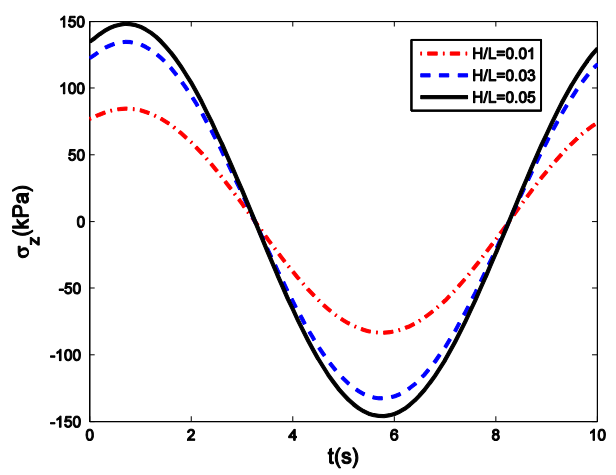

(c)Vertical effective normal stresses

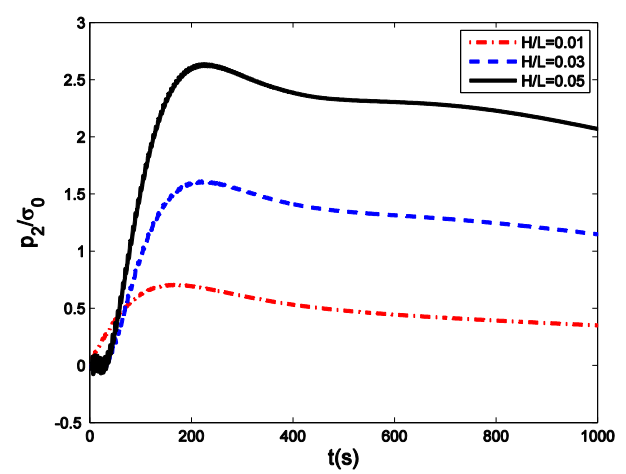

(b) Residual pore pressure

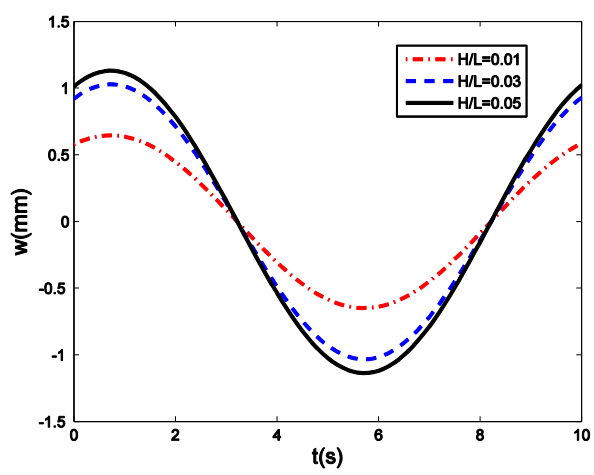

(d) Vertical soil displacement

Fig. (5). Effect of wave steepness $(H / L)$ on wave-seabed interaction at point $(x, y, z)=(0,0,-2) \mathrm{m}$. 


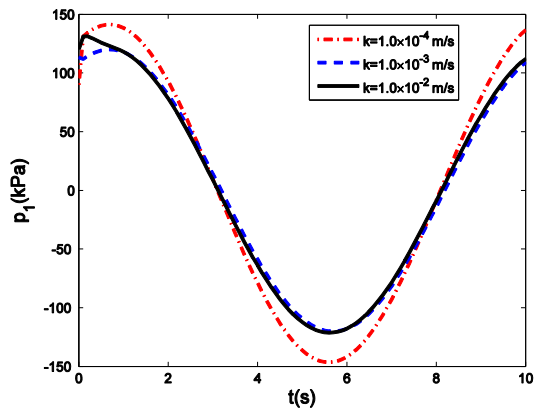

(a) Oscillatory pore pressure

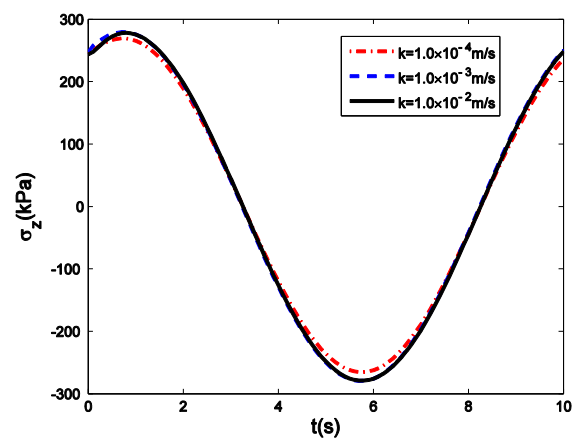

(c) Vertical effective normal stresses

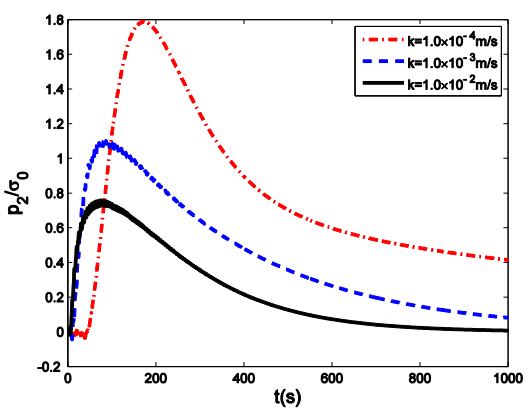

(b) Residual pore pressure

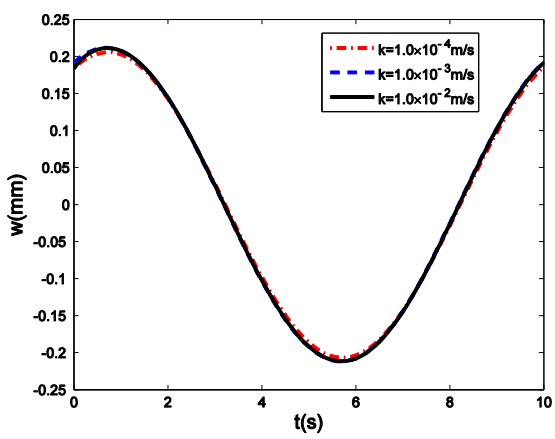

(d) Vertical soil displacement

Fig. (6). Effects of seabed permeability on wave-seabed interaction at point $(x, y, z)=(0,0,-2) m$.

pressure can accumulate to a higher value in a seabed with lower permeability than that with higher permeability.

It is common that the degree of saturation in marine sediment is very close to unity, implying almost fully

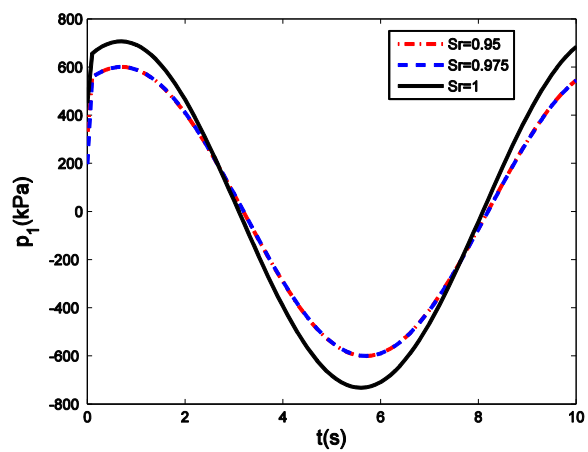

(a) Oscillatory pore pressure

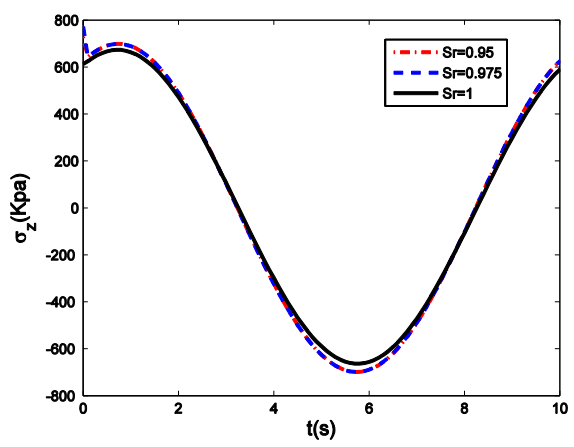

(c) Vertical effective normal stresses saturated [18]. As shown in Fig. (7), seabed saturation has barely any influence on vertical displacement and vertical stress, but it plays an important role in the development of residual pore pressures. Specifically, they are positively

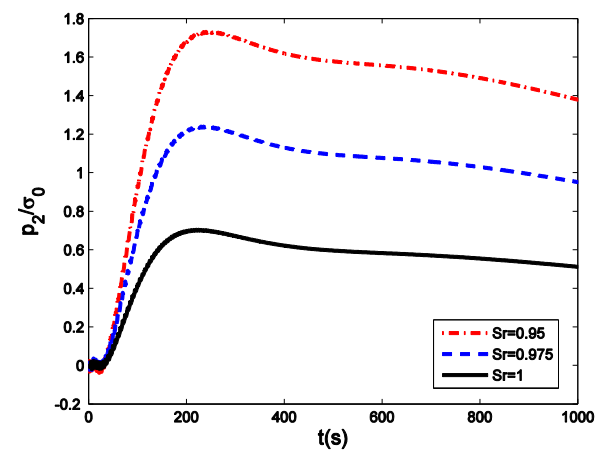

(b) Residual pore pressure

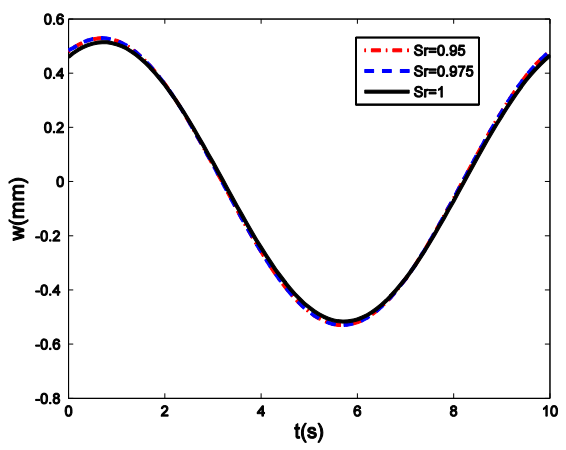

(d) Vertical soil displacement

Fig. (7). Effects of seafloor saturation on wave-seabed interaction at point $(x, y, z)=(0,0,-2) m$. 


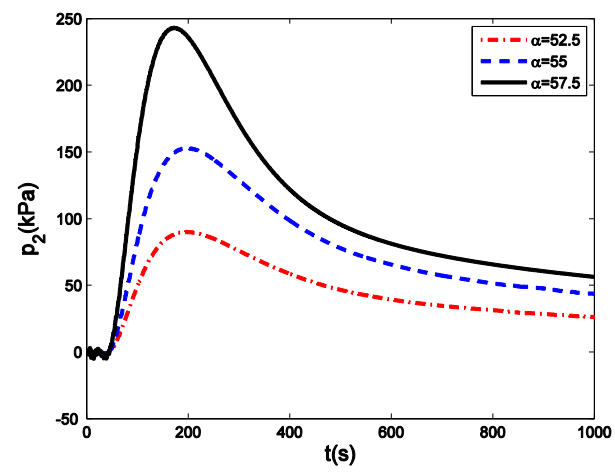

(a) Plastic parameter $\alpha$

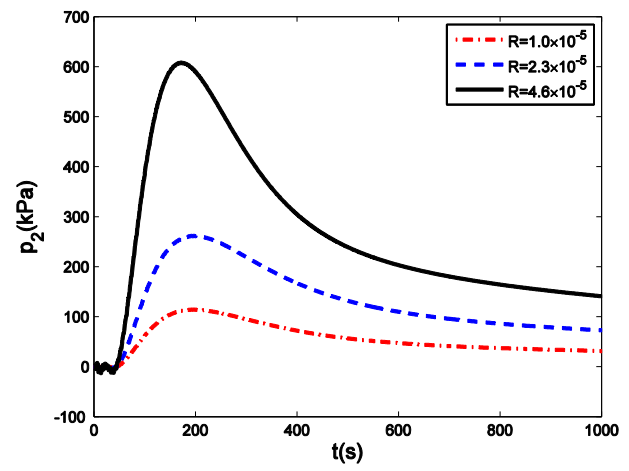

(b) Plastic parameter $\mathrm{R}$

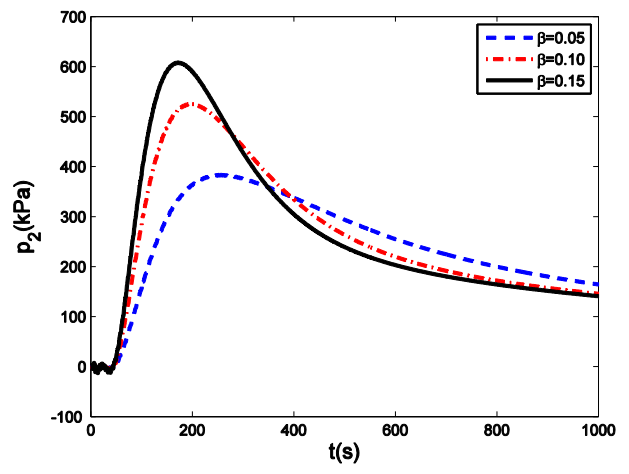

(c) Plastic parameter $\beta$

Fig. (8). Effects of residual parameters on wave-seabed interaction at point $(x, y, z)=(0,0,-2) \mathrm{m}$.

correlated, that is to say, residual pore pressure deceases while the seabed saturation is relatively high. Meanwhile, unlike the influence of permeability on residual pore pressure, on matter what the value of saturation is, the accumulation and dissipation process of residual pore pressure are at the same pace.

\subsection{Effects of Residual Parameters}

Three residual parameters $\alpha, \beta$, and $\mathrm{R}$ are inclusively concerned with residual pore water pressure. Explicitly, they are associated with the accumulation process of plastic volumetric strain. $\beta$ is a material parameter dominating the velocity of accumulation of plastic volumetric strain $\varepsilon_{2}$, and $\varepsilon_{2}^{\infty}$ represents the value of plastic volumetric strain when loading cycle ultimately approaches infinity. Based on the Sekiguchi's previous conclusion [11], $\varepsilon_{2}^{\infty}$ is interconnected with material parameter $\alpha$ and $\mathrm{R}$, in the form of $\varepsilon_{2}^{\infty}=$ $\mathrm{R}\left(\mathrm{e}^{\alpha \phi}\right)$, where $\phi$ is the cyclic stress ratio. As presented in Fig. (8), they are positively connected in the cumulating stage. That is, the wave-induced residual pore water pressure increases with plastic parameters $\alpha, \beta$ and $\mathrm{R}$ in magnitude. While in the dissipating process, residual pore water pressure increases with parameter $\alpha$ and $\mathrm{R}$, but decreases with plastic parameter $\beta$, which is a material parameter dominating the velocity of accumulation of plastic volumetric strain. It might be an interesting and meaningful discovery leading a new way to research the mechanism of residual pore water pressure dissipation, since parameter $\beta$ affects residual pore pressure differently as plastic parameter $\alpha$ and parameter $\mathrm{R}$ does.

\section{CONCLUSION}

In this study, a 3D poro-elastoplastic model in marine sediments is established to explore the short-crested waveinduced pore water pressures. After theoretical expansion of seabed elasticity and plasticity from $2 \mathrm{D}$ to $3 \mathrm{D}$, the present modeling results with previous wave experiment was validated, and it receives a fairly ideal consistency. Based on numerical examples presented, the following conclusions can be drawn.

1) The wave obliquity has some influence on the waveinduced soil response. For example, when a short-crested wave has a 45 degree incident angel, it would have a more mild interaction with seabed surface, which might greatly lower the crisis of liquefaction failure of structures. Wave steepness $(\mathrm{H} / \mathrm{L})$ is one of key factors in the determination of the wave-induced liquefaction potential. Basically, residual liquefaction will occur when the wave steepness increases to a certain value.

2) Both soil permeability and saturation have less impact on the vertical soil displacements and effective normal stresses, while it significantly affects the pore water pressures. Practically speaking, residual pore water pressure can accumulate to higher value in a seabed with low permeability. 
3) Residual parameters are generally positively connected with residual pore water pressure during both accumulating and dissipating process except that residual pore water pressure and plastic parameter $\beta$ are negatively interrelated in the dissipation stage, which might be a new route to reveal the mechanism of residual pore water pressure dissipation stage.

\section{CONFLICT OF INTEREST}

The authors confirm that this article content has no conflict of interest.

\section{ACKNOWLEDGMENTS}

The authors gratefully acknowledge the support of National Natural Science Foundation of China (NSFC) Grant \# 41176076 (China).

\section{REFERENCES}

[1] W. Smith, and A. D. Goldon, "Large breakwater toe failures", Journal of the Waterway, Harbor, Coastal Engineering Division, ASCE, vol. 109, no. 2, pp. 253-255, 1983.

[2] J. T. Christian, P. K. Taylor, J. K. C. Yen, and D. R. Erali, "Large diameter underwater pipeline for nuclear power plant designed against soil liquefaction", In: Proceeding of Offshore Technology Conference, 1974, pp. 597-606.

[3] H. Lundgren, J. H. C. Lindhardt, and C. J. Romold, "Stability of breakwaters on poros foundation", In: Proceeding of $12^{\text {th }}$ International Conference on Soil and Foundation Engineering, 1989, pp. 451-454.

[4] D. S. Jeng, Porous Models for Wave-seabed Interactions, Springer Heidelberg, 2013.
[5] O. S. Madsen, "Wave-induced pore pressure and effective stresses in porous bed", Géotechnique, vol. 28, no. 4, pp. 377-393, 1978.

[6] H. B. Seed, and M. S. Rahman, "Wave-induced pore pressure in relation to ocean floor instability of cohesion less soils", Marine Geotechnolgy, vol. 3, no. 2, pp. 123-150, 1978.

[7] Y. H. Maeno, and T. Hasegawa, "Evaluation of wave-induced pore pressure in sand layer by wave steepness", Coastal Engineering in Japan, vol. 28, pp. 31-44, 1985.

[8] Y. T. Tsui, and S. C. Helfrich, "Wave-induced pore pressures in submerged and layer", Journal of Geotechnical Engineering, ASCE, vol. 109, no. 4, pp. 603-618, 1983.

[9] M. A. Biot, "General theory of three-dimensional consolidation", Journal of Applied Physics, vol. 26, no. 2, pp.155-164, 1941.

[10] D. J. Pickering, "Anisotropic elastic parameters for soil", Géotechnique, vol. 20, no. 3, pp. 271-276, 1970.

[11] H. Sekiguchi, K. Kita, and O. Okamoto, "Response of poroealstoplastic beds to standing waves", Soils and Foundations, vol. 35, no. 3, pp. 33-42, 1995.

[12] S. Sassa, and H. Sekiguchi, "Wave-induced liquefaction of beds of sand in a centrifuge", Géotechnique, vol. 49, No. 5, pp. 21-638, 2001.

[13] C. Liao, H. Zhao, and D. -S. Jeng, "Poro-Elastoplastic Model for the Wave-induced Liquefaction", Journal of Offshore Mechanics and Arctic Engineering, ASME (in press).

[14] T. Yamamoto, H. Koning, H. Sellmeijer, and E. V. Hijum, "On the response of a poro-elastic bed to water waves", Journal of Fluid Mechanics, vol. 7, no. 1, 193-206, 1978.

[15] J. R. C. Hsu, Y. Tsuchiya, and R. Sylvester, "Third-order approximation to short-crested waves", Journal of Fluid Mechanics, vol. 90, pp. 179-196, 1979.

[16] M. Sumer, J. Fredsøe, S. Christensen, and M. L. Lind, "Sinking/ floatation of pipelines and other objects in liquefied soil under waves", Coastal Engineering, vol. 38, pp. 53-90, 1999.

[17] J. D. Fenton, "Wave forces on vertical walls", Journal of the Waterway, Harbor, Coastal Engineering Division, ASCE, vol. 114, no. 4, pp. 693-718, 1985.

[18] S. Pietruszczak, and G. N. Pande, "Constitutive relations for partially saturated soils containing gas inclusions", Journal of Geotechnical Engineering, ASCE, vol. 122, no. 1, pp. 50-59, 1996.

(C) Wong et al.; Licensee Bentham Open.

This is an open access article licensed under the terms of the Creative Commons Attribution Non-Commercial License (http://creativecommons.org/licenses/ by-nc/3.0/) which permits unrestricted, non-commercial use, distribution and reproduction in any medium, provided the work is properly cited. 